\title{
A Game Theoretical Network-Assisted User-Centric Design for Resource Allocation in 5G Heterogeneous Networks
}

\author{
Hamnah Munir*, Syed Ali Hassan*, Haris Pervaiz ${ }^{\dagger}$ and Qiang $\mathrm{Ni}^{\dagger}$ \\ *School of Electrical Engineering \& Computer Science (SEECS), National University of Sciences \& Technology \\ (NUST), Islamabad, Pakistan 44000, \{14mseehmunir, ali.hassan $\} @$ seecs.edu.pk \\ ${ }^{\dagger}$ School of Computing \& Communications, Lancaster University, UK \\ $\{$ h.pervaiz, q.ni\}@lancaster.ac.uk
}

\begin{abstract}
For the past few years, 5G heterogeneous networks (HetNets) have gain phenomenal attention in the wireless industry. In this paper, we propose a hierarchical game theoretical framework for the optimal resource allocation on the uplink of a heterogeneous network with femtocells overlaid on the edge of a macrocell. In the first game, the femtocell access points (FAPs) play a non-cooperative game to choose their access policy between open and closed in order to maximize the rate of their home subscribers. The second game of the algorithm allows macrocell user equipments (MUEs) to decide their connectivity between the FAPs and the macrocell base station (MBS) with the goal of maximizing their rates and the overall network performance; thereby, distributing intelligence and control to the users. The FAPs and the MUEs are the players of two different games that strategically decide their policies in an ordered fashion. Simulation results show that this hierarchical game approach with network-assisted user-centric design offers a significant improvement in terms of the performance of HetNets relative to an closed and only network-centric access policy schemes.
\end{abstract}

Index Terms-Heterogeneous network, game theory, Nash equilibrium, femtocell, user-centric, sum-rate.

\section{INTRODUCTION}

With the drastic increase in wireless data traffic, the demand for higher data rates has become a key necessity for the next generation mobile network. Heterogeneous networks (HetNets), consisting of macrocells, picocells and femtocells, have gained much momentum as a solution to this problem in fifth generation (5G). While improving overall network performance, it faces many challenges including network modeling, radio resource management and energy efficiency and several existing works have addressed these challenges [1].

The deployment of femtocells helps in increasing the sumrate of the network but makes interference and centralized control a challenging issue [2]. A considerable amount of literature is available to address this concern of interference as seen in [3] and the references therein. To reduce monitoring complexity associated with centralized control for decision making, user-centric schemes have drawn major attention. In a user-centric scheme, user is on top of all that makes decision with or without network-assistance. User-centric scheme focuses on the interest of the users and requires less computa- tional complexity whereas network-centric scheme can make more informed decisions at the cost of monitoring overhead. Thus, a fusion of the aforementioned schemes can generate interesting results [4].

The femto access points (FAPs) can operate in different modes: closed, open and hybrid [5]. In closed access scheme, resource sharing is not allowed and FAPs dedicate all of their resources to their home subscribers. Whereas in open access scheme, FAPs share their resources with the macrocell users in order to avoid interference and to enhance the network performance. The hybrid access policy puts a limit on the resource allocation to macrocell users [6]. The selection of access policy on the uplink is a tradeoff between interference avoidance and saving resources and has a significant impact on the performance of the network. Several existing works used the game theoretical models to optimize the performance of femtocells in the HetNets [7].

In this paper, we present a hierarchical game theoretical framework consisting of two sub-games for resource allocation to optimize the sum-rate of a heterogeneous network. This scheme starts by modeling the FAPs preferred access policies to optimize the performance of their registered users in the first game, given the state of the network. The main focus of this part is to analyze the conflicting interests of the FAPs in the selection of their optimized access policies. The second game uses user-centric approach by allowing the macrocell users to finalize their association in order to maximize their interest while keeping in view the network performance. To solve this hierarchical game framework, we devise a distributed scheme which always reaches a pure strategy Nash equilibrium (PSNE). The coalition of two games optimises the data rates for macrocell users and femtocell users, at the expense of increased complexity of the game problem. Simulations have shown that this proposed scheme outperforms the networkcentric scheme by a huge margin.

The rest of this paper is organized as follows. In Section II, we present the system model of the proposed hierarchical game framework. In Section III, we discuss the proposed algorithm for network-assisted user-centric resource allocation. Section VI shows the simulation results and Section V concludes the paper along with the future work. 


\section{SySTEM MODEL}

Consider the uplink of a single cell heterogeneous network having $M$ femtocell access points (FAPs) overlaid on a macrocell, as shown fig. in 1, having $N$ macrocell user equipments $(M U E s)$. Let $\mathbf{M}=\{1,2, \ldots, M\}$ be the set of FAPs and $\mathbf{N}=\{1,2, \ldots, N\}$ be the set of macrocell users. We assume that a single femtocell user equipment (FUE) is connected to each FAP. The system bandwidth, $B$, is divided among FAPs in such a way that each FAP has $K$ subcarriers available, where $K=B / M$. This implies that the FUEs do not create interference on the uplink to other FAPs as different FAPs are allocated orthogonal bands using OFDMA. This can be done using other methods as explained in [8]. The same bandwidth, $B$, is also used by the macro base station $(M B S)$, where each MUE gets $L$ subcarriers $(L=B / N)$, which introduces cross-tier interference between the femtocells and the macrocell.

In this paper, we assume a Rayleigh fading channel with path loss. The channel between the $m^{\text {th }}$ FAP and $n^{\text {th }}$ MUE on $k^{t h}$ subcarrier is denoted by $h_{n m}[k]$, whereas the distance between them is denoted by $d_{n m}$. Similarly, the channel between the FUE and its corresponding FAP on the $k^{\text {th }}$ subcarrier be $h_{0 m}[k]$ and the distance between them is symbolized by $d_{0 m}$. Assume the channel between $n^{t h}$ MUE and MBS on $l^{\text {th }}$ subcarrier to be $h_{n b}[l]$ and the distance between them be $d_{n b}$. The channel between the FUE of $m^{t h}$ FAP and MBS is denoted by $h_{m b}[l]$ separated by the distance $d_{m b}$. The transmit power of $n^{t h}$ MUE is signified by $P_{n}$ and transmit power of each FUE by $P_{0}$. A Gaussian noise with zero mean and $\sigma^{2}$ variance is added to all subcarriers at all FAPs and MBS.

The signal-to-interference-plus-noise ratio (SINR) for the FUE at the $m^{t h}$ FAP is given by

$$
\operatorname{SINR}_{m}[k]=\frac{\mu_{m}[k]}{\sigma^{2}[k]+\Sigma_{n=1}^{N}\left(\prod_{i=1}^{M} \mathbb{1}_{\rho_{n}^{i}[k]=0}\right) \mu_{n}^{m}[k]},
$$

and the SINR for $n^{t h}$ MUE at $m^{t h}$ FAP is given by

$$
S I N R_{n, m}[k]=\frac{\left[1-\left(\prod_{i=1}^{M} \mathbb{1}_{\rho_{n}^{i}[k]=0}\right)\right] \mu_{n}^{m}[k]}{\sigma^{2}[k]+\Sigma_{n=1}^{N}\left(\prod_{i=1}^{M} \mathbb{1}_{\rho_{n}^{i}[k]=0}\right) \mu_{n}^{m}[k]},
$$

where $\mu_{m}[k]=\left(h_{0 m}[k]\right)^{2} P_{0} W\left(d_{0 m}\right)^{-\beta}$ is the received power from FUE at $m^{t h}$ FAP on $k^{t h}$ subcarrier and $\mu_{n}^{m}[k]=$ $\left(h_{n m}[k]\right)^{2} P_{n}\left(d_{n m}\right)^{-\alpha}$ is the received power from $n^{t h}$ MUE

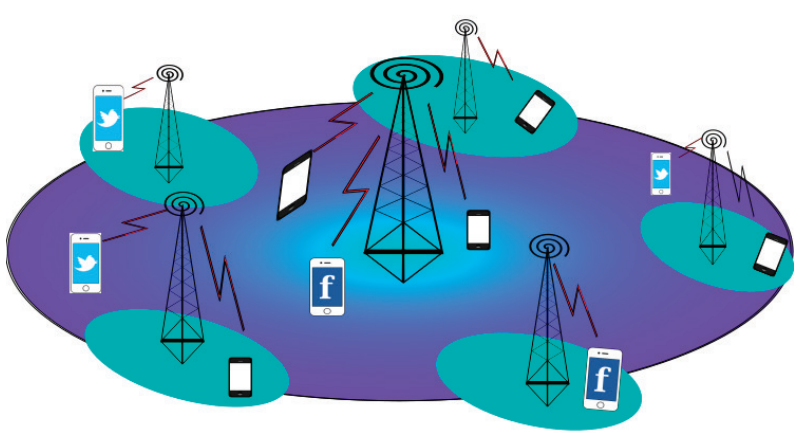

Fig. 1. A heterogeneous network with femtocells overlaid on a macrocell. at $m^{t h}$ FAP on the $k^{t h}$ subcarrier. The value $W<1$ is the wall penetration loss, $\alpha$ and $\beta$ are the path loss exponents.

Let $\rho_{n}^{m}[k] \in\{0,1\}$ signifies the connection of $n^{t h}$ MUE to $m^{t h}$ FAP on the $k^{t h}$ subcarrier. The connectivity between $n^{t h}$ MUE and $m^{\text {th }}$ FAP on the $k^{t h}$ subcarrier occurs when $\delta_{n}^{m}[k]=1$ and vice versa. The indicator function, $\mathbb{1}$, is defined as

$$
\mathbb{1}_{\{x\}}=\left\{\begin{array}{ll}
1 & x=0 \\
0 & x=1
\end{array} .\right.
$$

Here SINR of the $n^{\text {th }}$ MUE at MBS is expressed as

$$
S I N R_{n, b}[l]=\frac{\left(\prod_{i=1}^{M} \mathbb{1}_{\rho_{n}^{i}[l]=0}\right) \mu_{n}^{b}[l]}{\sigma^{2}[l]+\Sigma_{n=1}^{N}\left[1-\left(\prod_{i=1}^{M} \mathbb{1}_{\rho_{n}^{i}[l]=0}\right)\right] \mu_{n}^{b}[l]+\Sigma_{m=1}^{M} \mu_{m}^{b}[l]},
$$

where $\mu_{n}^{b}[l]=\left(h_{n b}[l]\right)^{2} P_{n}\left(d_{n b}\right)^{-\alpha}$ is the received power at MBS from $n^{t h}$ MUEs on $l^{t h}$ subcarrier and $\mu_{m}^{b}[l]=$ $\left(h_{m b}[l]\right)^{2} P_{0}\left(d_{m b}\right)^{-\alpha}$ is the received power at MBS from FUE of $m^{t h}$ FAP on $l^{\text {th }}$ subcarrier.

In our proposed approach, a hierarchical game consisting of two non-cooperative games is being played in a sequential order. In the first game, each FAP decides among open, closed and hybrid policy. Open access policy allows MUEs to connect to FAPs to reduce interference at the expense of resources. The closed access saves resources at the price of interference, whereas the hybrid policy is the trade off between interference and the cost of resources. This decision of FAPs depends on the interference from the MUEs and also on the choice of other FAPs, e.g., multiple FAPs cannot serve the same user as it would end up in resource wastage. Thus, the FAPs form a non-cooperative game with the goal of maximizing the rate of their FUEs by deciding its access policies. The strategy vector of FAP is the fraction of frequency band allocated to each MUE and utility function is the rate of its FUE, which can be written as

$$
\tilde{v}_{m}\left(\boldsymbol{\rho}_{m}, \boldsymbol{\rho}_{-m}\right)=\sum_{k=1}^{K}\left(\prod_{i=1}^{M} \mathbb{1}_{\rho_{n}^{i}[k]=0}\right) \log \left(1+S I N R_{m}[k]\right),
$$

where $\boldsymbol{\rho}_{m}=\left[\rho_{1, m}[1], . ., \rho_{N, m}[1], \rho_{1, m}[2], . ., \rho_{N, m}[K]\right]^{T}$ is strategy vector of $m$-th FAP, $\boldsymbol{\rho}_{-m}=$ $\left[\boldsymbol{\rho}_{1}^{T}, \ldots, \boldsymbol{\rho}_{m-1}^{T}, \boldsymbol{\rho}_{m+1}^{T}, \ldots, \boldsymbol{\rho}_{M}^{T}\right]^{T}$ shows the strategy vector of other FAPs and $[.]^{T}$ denotes the transpose operator.

In the other game, the MUEs re-evaluate their connectivity obtained from previous game, forming another noncooperative game with the goal of maximizing their rates without affecting the overall network performance. The strategy vectors of the MUEs are the fraction of band allocated to them by FAPs and MBS and the utilities are their rates. The utility function can be expressed as

$$
\begin{array}{r}
\tilde{v}_{n}\left(\boldsymbol{\rho}_{n}, \boldsymbol{\rho}_{-n}\right)=\sum_{k=1}^{K}\left[1-\left(\prod_{i=1}^{M} \mathbb{1}_{\rho_{n}^{i}[k]=0}\right)\right] \log \left(1+S I N R_{m}[k]\right)+ \\
\sum_{l=1}^{L}\left[\left(\prod_{i=1}^{M} \mathbb{1}_{\rho_{n}^{i}[l]=0}\right)\right] \log \left(1+S I N R_{b}[l]\right),
\end{array}
$$

where $\boldsymbol{\rho}_{n}=\left[\rho_{1, n}[1], . ., \rho_{M, n}[1], \rho_{1, n}[2], . ., \rho_{M, n}[2], . ., \rho_{M, n}[K]\right.$, 
$\left.\rho_{b, n}[1], \ldots, \rho_{b, n}[L]\right]^{T}$ is strategy vector of $n^{\text {th }}$ MUE and $\boldsymbol{\rho}_{-n}=\left[\boldsymbol{\rho}_{1}^{T}, . ., \boldsymbol{\rho}_{n-1}^{T}, \boldsymbol{\rho}_{n+1}^{T}, . ., \boldsymbol{\rho}_{N}^{T}\right]^{T}$ includes the strategy vectors of other MUEs.

The rate obtained by the MUE should not be less than a minimum acceptable rate, $R_{\min }$, which is fixed for all MUEs in the network. In case of connectivity between $m^{t h}$ FAP and $n^{\text {th }}$ MUE, this constraint is given by

$$
\begin{aligned}
& \left(1-\prod_{i=1}^{M} \mathbb{1}_{\rho_{n}^{i}[k]=0}\right) R_{\min } \leq \\
& \sum_{k=1}^{K} \rho_{n}^{m}[k] \log \left(1+\frac{\mu_{n}^{m}[k]}{\sigma^{2}[k]+\Sigma_{n=1}^{N}\left(\prod_{i=1}^{M} \mathbb{1}_{\rho_{n}^{i}[k]=0}\right) \mu_{n}^{m}[k]},\right.
\end{aligned}
$$

and for $n^{\text {th }}$ MUE connectivity with MBS, this constraint is written as

$$
\begin{aligned}
& \left(\prod_{i=1}^{M} \mathbb{1}_{\rho_{n}^{i}[l]=1}\right) R_{\min } \leq \\
& \sum_{l=1}^{L} \rho_{n}^{b}[l] \log \left(1+\frac{\mu_{n}^{b}[l]}{\sigma^{2}[l]+\sum_{m=1}^{M} \mu_{m}^{b}[l]+\sum_{n=1}^{N}\left[1-\left(\prod_{i=1}^{M} \mathbb{1}_{\rho_{n}^{i}[l]=0}\right)\right] \mu_{n}^{b}[l]} .\right.
\end{aligned}
$$

Now the strategy space for $m^{t h}$ FAP in the first phase is given as

$$
\tilde{\chi}_{m}=\left\{\boldsymbol{\rho}_{m}[k] \in(0,1)^{N K}: \sum_{n=1}^{N} \rho_{n}^{m}[k] \leq 1\right\} .
$$

The above constraint makes sure that not more than one MUE can be connected to $m^{\text {th }}$ FAP on $k^{t h}$ subcarrier. For given strategy vectors of other FAPs, we can define the optimization problem solved by $m^{\text {th }}$ FAP as

$$
\max _{\boldsymbol{\rho}_{m} \in \tilde{\chi}_{m}}\left(\boldsymbol{\rho}_{m}, \boldsymbol{\rho}_{-m}\right) \text {. }
$$

Strategy space of $n^{\text {th }}$ MUE for the second game is

$$
\tilde{\chi}_{n}=\left\{\boldsymbol{\rho}_{n}[l] \in(0,1)^{(M+1) L}:\left(\rho_{n}^{m}[l]+\rho_{n}^{b}[l]\right) \leq 1\right\} .
$$

This constraint ensures that the MUE cannot be connected to a FAP and MBS simultaneously. We can thus write the optimization problem as

$$
\max _{\boldsymbol{\rho}_{n} \in \tilde{\chi}_{n}}\left(\boldsymbol{\rho}_{n}, \boldsymbol{\rho}_{-n}\right) .
$$

We have solved the above games using Nash equilibrium. Nash equilibrium is attained by $\left(\boldsymbol{x}^{*}{ }_{i}, \boldsymbol{x}^{*}{ }_{-i}\right)$ when

$$
\tilde{f}_{i}\left(\boldsymbol{x}_{i}^{*}, \boldsymbol{x}_{-i}^{*}\right) \geq \tilde{f}_{i}\left(\boldsymbol{x}_{i}, \boldsymbol{x}_{-i}\right) ; \forall x_{i} \in \tilde{\chi}_{i},
$$

where $x_{i}$ represents the strategy vector of $i^{\text {th }}$ player with the utility function $f_{i}$.

\section{Proposed User Centric Resource Allocation SCHEME: A HIERARCHICAL GAME APPROACH}

We propose a distributed solution, which aims at maximizing the rate given to the users by optimizing the trade off between interference and the resources. The algorithm always reaches a pure strategy Nash equilibrium (PSNE) while achieving stable action profiles. It starts by allowing FAPs to select their strategies while knowing the strategies of other FAPs at any point in time, which is done using a parallel

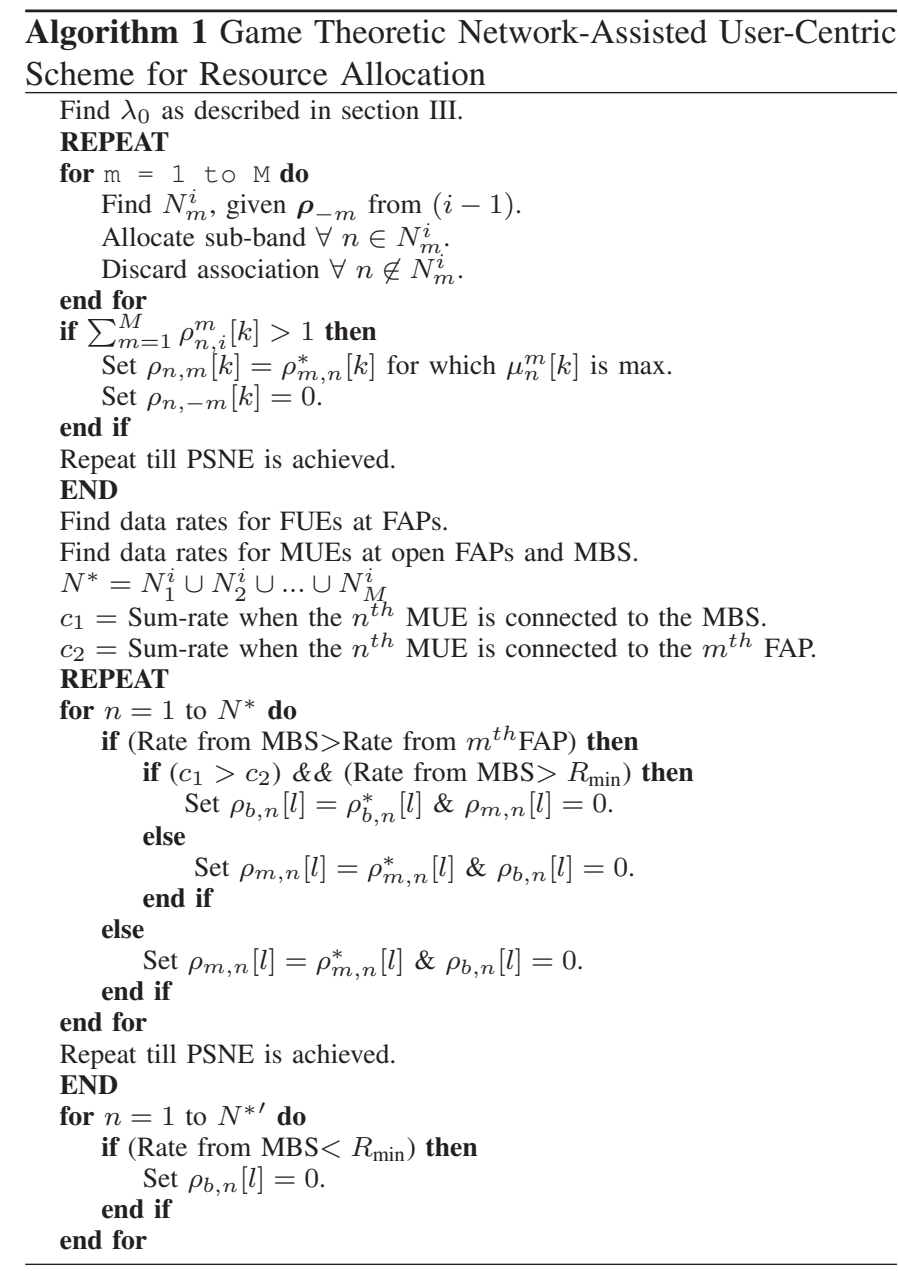

update technique. Using the information of other FAPs from the $(i-1)^{t h}$ iteration, each FAP selects its own strategy at the $i^{t h}$ iteration. The first step is to form an initial strategy vector $\lambda_{0}$, without seeking equilibrium. In this vector, optimal resources are allocated to all MUE while satisfying (6) using

$$
\lambda_{n}^{m}=\frac{R_{\min }}{\log \left(1+\frac{\mu_{n}^{m}}{\sigma^{2}}\right)} .
$$

After that, each FAP explores the favorable set of MUEs $\left(N_{m}^{i}\right)$ in each iteration, given the strategies of other FAPs from $(i-1)^{t h}$ iteration. The selection of $N_{m}^{i}$ ( $N_{m}^{i}$ can be empty) is done in order to maximize the rates of FUEs (utility function of the FAPs). In case of open access, each FAP needs to optimize the selection of $N_{m}^{i}$ by checking the utility from servicing a certain set of MUEs. To avoid complexity, the FAP could find optimal set of MUEs with the help of greedy algorithm as used in [9] rather than testing all possible combinations of MUEs. Greedy algorithm helps FAPs by finding highly interfering MUEs. Each iteration ends with the assurance that multiple FAPs are not allocating resources to a single MUE as it would result in the waste of resources. The connectivity between FAPs and MUEs ensures the best interest of the users of FAPs. These iterations continues until convergence, which can also be achieved using other schemes, such as in [10].

After maximizing the rates of FUEs, MUEs play the next game to maximize their rates using user-centric approach. 
MUEs which are connected to FAPs, as a result of previous game, examine the rates they are getting from FAP and MBS. MUEs stay connected to FAPs if the utility is greater for that case. If the rate that the MUE is getting from the MBS is greater, then the sum-rate is calculated for both cases with MUE connected to MBS and with FAP. Each MUE opts for the case where system is not affected and it gets the rate greater than a defined threshold of $R_{\min }$. If the constraint of $R_{\min }$ is not met, the particular MUE goes into outage. At the end of this game, each MUE ensures that it is not connected to FAP and MBS simultaneously, thus saving resources. The above steps are continued until all MUEs, which were previously connected to FAPs, finalize their strategies in the best interest of the network and themselves.

\section{Simulation Results}

In this section, we present the numerical results of our proposed algorithm with respect to various network parameters. We consider a cell of $1000 \mathrm{~m}$ radius where the FAPs and the MUEs are uniformly scattered over the area. The FUEs and the MUEs are assured to have same transmit power of 0.2 W. The path loss exponent $\alpha=2, \beta=2.5$ and the wall penetration loss $W=0.5$ is assigned. The distance between each FAP and its corresponding FUE is $1 \mathrm{~m}$. It is assumed for simplicity that each FAP has one FUE. The noise variance is set to $\sigma^{2}=10^{-14}$. The system bandwidth, $B=10 \mathrm{MHz}$ and the minimum acceptable date rate for the MUEs is $500 \mathrm{kbps}$ unless rated otherwise.

The improvement in the sum-rate for the proposed approach relative to all closed and network-centric scheme for $N=7$ is shown in Table I . It can thus be concluded that our proposed scheme outperforms both schemes by a significant margin. This distributed algorithm ensures the welfare of all the users by optimizing their utility functions.

We have compared our proposed scheme with two other schemes. The first comparison of our proposed scheme is done with an all-closed access policy scheme, where all the FAPs have adopted a closed access that results in connecting all the MUEs to the MBS. On the other hand, the second comparison is with the network-centric optimized scheme. This scheme allows FAPs and MBS to decide the connectivity of their users. Hence the central entity reserves all the control. In our proposed scheme, we have merged the network-centric approach with the user-centric approach by spreading the control and intelligence in the network rather than keeping it to the central entity. This user-centric scheme not only overtakes the network-centric scheme in terms of performance but also offloads the complex computation from MBS and distributes it to the network, thus requiring less computational and monitoring complexity.

Fig. 2 shows a comparison of the achieved sum-rate of the proposed scheme with closed access scheme and networkcentric optimized scheme. We can see that as $M$ increases, the sum-rate increases for the proposed scheme. This is because the likelihood of the FAPs playing open access increases with an increase in the number of FAPs, which in return service the interfering MUEs; thus improving the performance of the
TABLE I

SUM-RATE COMPARISON $(N=7)$.

\begin{tabular}{|c|c|c|}
\hline $\begin{array}{c}\text { No. } \\
\text { of } \\
\text { FAPs } \\
(\mathrm{M})\end{array}$ & $\begin{array}{c}\text { Improvement } \\
\text { relative to all } \\
\text { closed } \\
\text { scheme } \\
\text { access }\end{array}$ & $\begin{array}{c}\text { Improvement } \\
\text { relative to } \\
\text { network- } \\
\text { centric } \\
\text { optimized } \\
\text { scheme }\end{array}$ \\
\hline 2 & $18.52 \%$ & $17.07 \%$ \\
10 & $48.43 \%$ & $36.36 \%$ \\
18 & $60.53 \%$ & $41.86 \%$ \\
24 & $65.79 \%$ & $43.83 \%$ \\
\hline
\end{tabular}

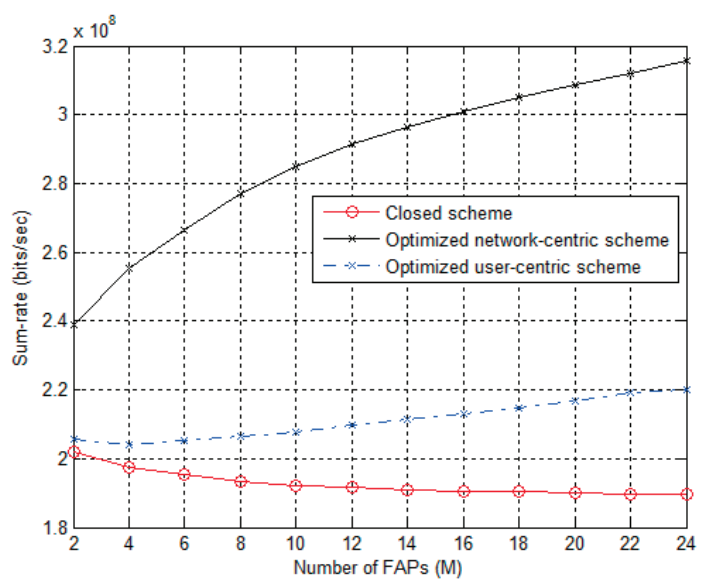

Fig. 2. Sum-rate of an all closed scheme, optimized network-centric scheme and proposed optimised user centric scheme with varying number of FAPs and $\mathrm{N}=7$

system and decreasing the outage probability. The same trend of sum-rate is followed in the network-centric scheme but usercentric scheme yields a significant improvement in terms of utilities. In the case of all closed access scheme, the sumrate almost remains constant, although the number of FAPs increases. This is due to the fact that as the density of FAPs increases in the network, the MUEs appear closer to them resulting in increased interference. This, in turn, decreases the data rates of the FUEs and also forces the MUEs to go in as seen in Fig. 3. The outage probability trend is same for both user-centric and network-centric schemes as demonstrated in Fig. 3, however, the proposed approach performs better in terms of achieved data rate. Thus, we can say that our scheme is as fair as network-centric though more capacity oriented.

Fig. 4 shows the comparison of sum-rate for an all closed scheme, optimized network-centric scheme and proposed scheme against different minimum rate requirements.the percentage of users in outage for the proposed scheme and the network-centric scheme is same although the sum-rate for proposed scheme is better as described earlier. This difference in sum-rate decreases as the minimum required rate increases because the condition of $R_{\min }$ is not satisfied and MUEs do not participate in the optimization of sum-rate. However, for increased number of users, this difference will again prevail. 


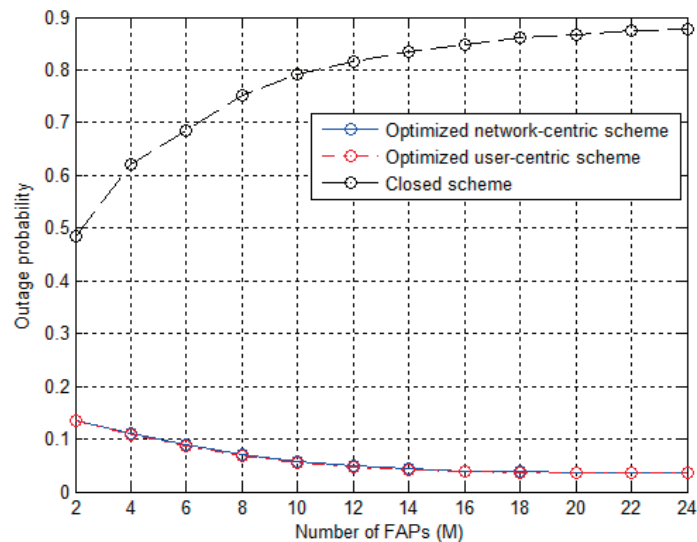

Fig. 3. Outage probability of an all closed scheme, optimized network-centric scheme and proposed scheme for varying number of FAPs with $\mathrm{N}=7$.

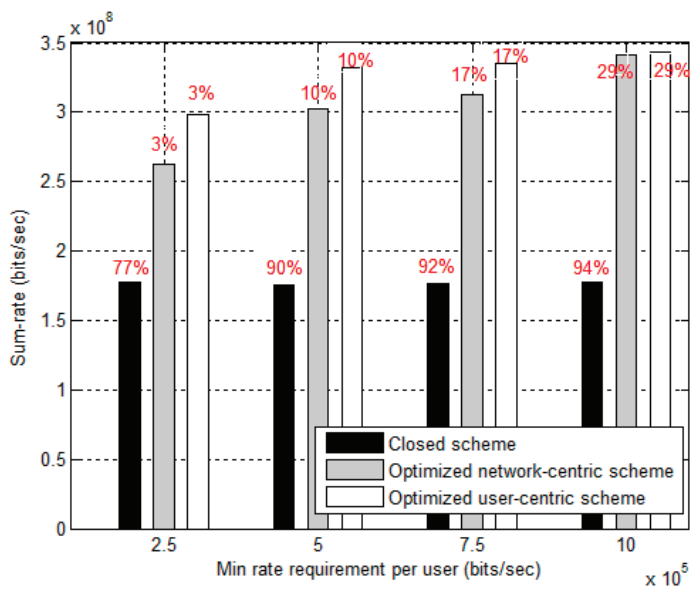

Fig. 4. Sum-rate of an all closed scheme, optimized network-centric scheme and proposed scheme vs the minimum rate requirement for $\mathrm{N}=12$ and $\mathrm{M}=10$ with outage (shown in \% at the top of each bar).

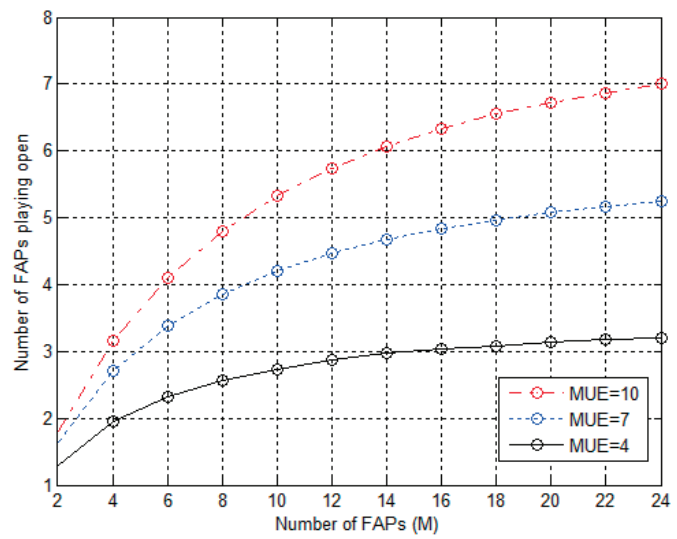

Fig. 5. Number of FAPs playing open access versus the varying number of FAPs.

In case of all closed scheme, the sum-rate remains constant while the outage percentage increases. This trends shows that for small value of minimum required rate e.g. $250 \mathrm{kbps}$, lesser users are in outage while for high value of rate requirement, e.g., $1 \mathrm{Mbps}$, more users are in outage, however, each serviced user is getting four times the data rate than the previous case. Hence the overall rate attained remains the same.

In Fig. 5, the number of FAPs playing open access policy are shown for our proposed approach. We can see that as $M$ increases, the number of open FAPs starts increasing to service the MUEs till it reaches a saturation point. This trend shows that as the number of FAPs start getting larger than the MUEs, additional FAPs should not play open to save their resources. We can observe that the number of FAPs playing open increases when $M \leq 6$ for a total of $N=7$. However, for $N=10$ this increasing trend continues for $M \leq 8$ and this number increases for $M \leq 3$ in case of $N=4$.

\section{Conclusions and Future Work}

A hierarchical game-theoretic framework for resource allocation is proposed in this paper, which allows the FAPs to strategically decide between the conflicting access modes while optimizing their allocated resources. It also enables the MUEs to decide their connectivity while acquiring their stable action profiles. The main focus of the players is to optimize the tradeoff between reducing interference and the cost of allocated resources. This hierarchical game framework optimizes the data rates of the FUEs and the MUEs while achieving the Nash equilibrium. We have applied low complexity user-centric distributed approach to improve the performance of the network and the simulation results have proved that the proposed algorithm significantly outperforms the network-centric scheme. In future, we will investigate the optimal power allocation for network entities which may further improve the performance of the proposed scheme.

\section{REFERENCES}

[1] H. Pervaiz, L. Musavian, Q. Ni, and Z. Ding, "Energy and spectrum efficient transmission techniques under QoS constraints toward green heterogeneous networks," IEEE Access, vol. 3, pp. 1655-1671, 2015.

[2] V. Chandrasekhar, J. G. Andrews, and A. Gatherer,"Femtocell networks: a survey," IEEE Commun. Mag., vol. 46, no. 9, pp. 59-67, 2008

[3] D. L'opez-P'erez, A. Valcarce, G. De La Roche, and J. Zhang, "OFDMA femtocells: A roadmap on interference avoidance," IEEE Commun. Mag., vol. 47, no. 9, pp. 41-48, 2009.

[4] S. E. Elayoubi, E. Altman, M. Haddad, and Z. Altman, "A hybrid decision approach for the association problem in heterogeneous networks," IEEE INFOCOM, pp. 1-5, 2010.

[5] D. Choi, P. Monajemi, S. Kang, and J. Villasenor, "Dealing with loud neighbors: The benefits and tradeoffs of adaptive femtocell access," Proc IEEE Global Telecommun. Conf. (GLOBECOM), pp. 1-5, Dec. 2008.

[6] H.-S. Jo, P. Xia, and J. G. Andrews, "Open, closed, and shared access femtocells in the downlink," EURASIP Journal on Wireless Commun. and Networking, vol. 2012, no. 1, pp. 1-16, 2012.

[7] X. Kang, R. Zhang, and M. Motani, "Price-based resource allocation for spectrum-sharing femtocell networks: A stackelberg game approach," IEEE Journal on Selected Areas in Communications, vol. 30, no. 3, pp. 538-549, 2012.

[8] N. Saquib, E. Hossain, L. B. Le, and D. I. Kim, "Interference management in OFDMA femtocell networks: Issues and approaches," IEEE Wireless Commun., vol. 19, no. 3, pp. 86-95, 2012.

[9] P. Xia, V. Chandrasekhar, and J. G. Andrews, "Open vs. closed access femtocells in the uplink," IEEE Trans. Wireless Commun., vol. 9, no. 12, pp. 3798-3809, 2010.

[10] T. Basar, G. J. Olsder, G. Clsder, T. Basar, T. Baser, and G. J. O 1sder, "Dynamic Noncooperative Game Theory," 2nd ed. Philadelphia, PA: SIAM, 1999. 